\title{
Fallot tetralogy course not corrected during pregnancy. about a case
}

\begin{abstract}
Fallot tetralogy is the most prevalent cyanogenic congenital heart disease. After one year of age, representing $10 \%$ of congenital heart defects and $75 \%$ of cyanotic heart defects. It is characterized by misalignment (anterior, superior, and right of the infundibular septum), causing the four known elements of pulmonary artery infundibular stenosis, aortic thrust, and ventricular septal defect, and hypertrophy of the right ventricle. Pregnancy in nonoperated women has an associated maternal mortality rate of $3 \%$ to $12 \%$, a perinatal loss rate (up to $30 \%$ ). Women who become pregnant are exposed to hemodynamic stress and a gradual increase in the severity of pulmonary stenosis, exacerbating symptoms, and increased cyanosis. Below we present the case of a patient diagnosed with tetralogy of Fallot diagnosed in adolescence without surgical correction, due to lack of financial resources who reaches the end of pregnancy, without adequate prenatal control, and exacerbation of symptoms in the last trimester, due to which interrupts pregnancy via the abdomen, with a favorable evolution during the puerperium.
\end{abstract}

Keywords: tetralogy of Fallot, pregnancy, congenital heart disease
Volume 10 Issue 4 - 2020

\author{
Sindy Arisbeth Salas-Villarruel,' Sandra \\ Pérez-Silva,' María Valeria Jiménez-Báez, \\ Tomás-Alvarado-Eduardo, ${ }^{3}$ Moises Alejandro \\ Toledo-Pensamiento ${ }^{4}$ \\ 'Department of gynecology, Regional General Hospital No. 17, \\ Mexican Institute of Social Security, Mexico \\ 2Planning Coordination and Institutional Liaison, Head of \\ Medical Benefits Services, Mexico \\ ${ }^{3}$ Department of Pediatric Cardiology, Regional General Hospital \\ No. 17, Mexico
}

${ }^{4}$ Director of Regional General Hospital No. I, Mexico

Correspondence: María Valeria Jiménez Báez, Head of Medical Benefits ServicesAv. Politecnico Nacional S/n c.p. 775 I0, Mexico, Tel 9988742354 ,

Email valeria.jimenezb@gmail.com

Received: July 07, 2020 | Published: July 20, 2020

\section{Introduction}

Tetralogy of Fallot (F.T.) is the most prevalent cyanogenic congenital heart disease after one year of age, characterized by misalignment (anterior, superior, and right of the infundibular septum), thus causing the four known elements: infundibular stenosis of the pulmonary artery, aortic thrust, communication and ventricular hypertrophy of the right ventricle.

Incidence of 5 to $8 \%$ of all congenital heart defects and about half of these cases occur in women. Heart diseases being the leading cause of indirect maternal mortality during pregnancy. ${ }^{2,3}$

The vast majority of carriers of this defect may have survival almost equal to that of the general population. Although the F.T. pregnancy's delivery without to have corrective surgery. ${ }^{4}$

The women with congenital heart disease have hemodynamics modifications during pregnancy and labor periods. These are poorly tolerating and produce serious complications, including maternal mortality secondary to pulmonary thromboembolism. ${ }^{5}$ Incrementing fetal risk disorders: miscarriage, intrauterine growth restriction, prematurity, perinatal mortality, and congenital heart disease in the fetus. ${ }^{6-9}$

The pathophysiological characteristics depend on the degree of obstruction of the right ventricular outflow tract. If the obstruction is severe, the passage of non-oxygenated blood through non restrictive ventricular septal defect to the aorta is increased, with increased cyanosis. In cases where the obstruction is slight, also called Pink Fallot, most blood flow from the right ventricle passes to the pulmonary artery, and cyanosis is minimal and behaves like a ventricular septal defect with a left-to-right shunt. ${ }^{1,10}$

The clinical behavior of adults and pediatrics is entirely different, highlighting the previous radiological study with a regular or slightly increased vascular pattern. On the electrocardiogram, a block of the right bundle branch of His founding $50 \%$ of patients. ${ }^{3}$

The maternal and fetal risk of pregnancy for this population will depend on the anatomical and physiological classification that evaluating using cardiovascular medical history, electrocardiogram, and transthoracic echocardiogram.

The purpose of this study is to report the case of a patient with a diagnosis of tetralogy of Fallot diagnosed in adolescence without surgical correction whose resolution is performed in the last trimester through the abdominal route, with a favorable evolution during the puerperium. A review of the scientific literature and show current management news.

\section{Presentation of the case}

The 28-year-old female from Veracruz, resident of Quintana Roo, married, Catholic, high school education, cleaning occupation. Gesta 1, FUM 10-11-2019, with approximately 11 prenatal consultations in private and institutional settings.

He entered the Regional General Hospital No. 17 Cancún Quintana Roo, referred from the UMF with diagnosis: Pregnancy of 33.4 SDG due to FUM / Tetralogy of Fallot not corrected. Diagnosed at three months of age with bronchial asthma, due to the presence of cyanotic cries when crying, persistent acrocyanosis, and dyspnea with great efforts, she underwent cardiac catheterization at 19 years of age, where apparent tetralogy of Fallot was determined, with the option of surgical treatment, which not done due to a lack of resources. In 2019, due to the desire for pregnancy, she went to the cardiology service for evaluation, performing an echocardiogram with the following result, an undilated left ventricle, severe dilation of the aortic root with slight insufficiency, without contraindication to pregnancy.

In May 2020, as part of the medical approach, an evaluation 
was sent to assess the evolution of heart disease in pregnancy with echocardiography, reporting the following: non-hypertrophic left ventricle, not dilated, LVEF 63\%, non-hypertrophic right ventricle, undilated without short circuit, slightly dilated right atrium, aorta thrust without obstruction, severe aortic root dilation with slight insufficiency, without pulmonary hypertension.

She refers to starting with dyspnea of medium efforts during the third trimester of gestation, which partially improves at rest, however during the last ten days before her hospitalization with exacerbation of dyspnea, making small efforts and edema of the lower extremities.

Upon admission, neurologically integrated, generalized pallor of skin and integuments, hydrated oral mucosa, both hemithorax with audible vesicular murmur without aggregates, heart sounds, goo. Holosystolic murmur intensity grade $\mathrm{V}$ in the pulmonary focus predominantly. Palpation globose abdomen with pregnant uterus fundus $28 \mathrm{~cm}$, cephalic presentation, longitudinal situation, left dorsum, FCF 140x, posterior cervical, vaginal touch closed, length cervical $3 \mathrm{~cm}$, full limbs, symmetrical, distal acrocyanosis, fingers on drumsticks. (Figure 1). Oxigen saturation 92\%, no edema, pulses without alterations capillary, refill 2 seconds. Upon admission laboratories and cabinet, clinical labs with the following result.

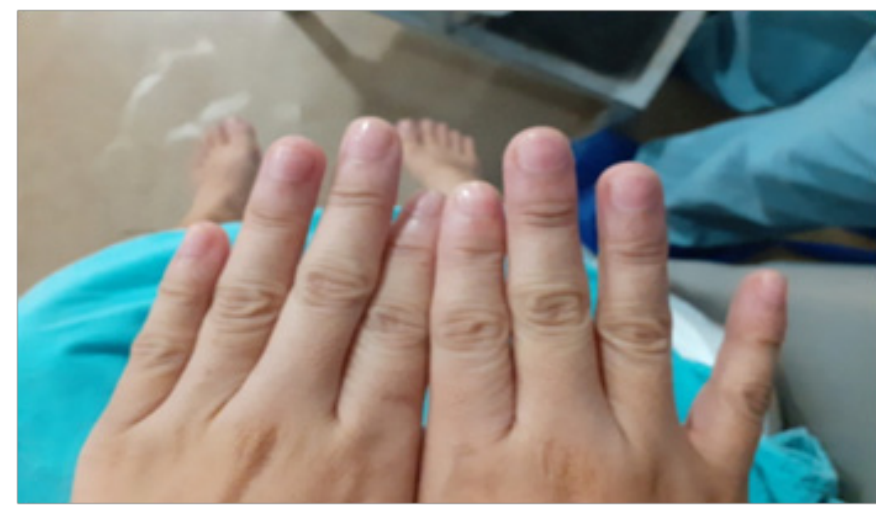

Figure I Photograph after birth where can see drumstick fingers.

Acid-base balance without apparent complications ph: 7.41, pO2 43, pCO2 31, HCO3: 19, laboratories (Table 1), Tele chest (figure 2 ), electrocardiogram (figure 3), echocardiogram (figure 4). The abdominal route is interrupted under epidural anesthesia. Pregnancy with the following findings obtaining on 04-03-2020 at 11:29 hrs. R.N. female weight $2120 \mathrm{~g}$, size $44 \mathrm{~cm}$. APGAR 5/7, Capurro 34 SDG. After a surgical event, she admits to the ICU as multidisciplinary management; oxygen supplementation starts, and the latter prophylactic low-molecular-weight heparin, after four days of ICU treatment with favorable evolution, is admitted to the gynecology floor to begin cardiac rehabilitation and continuous monitoring, thus progressing correct deciding discharge from the service.

\section{Discussion}

Heard disease in pregnancy is a severe problem since it is the leading cause of no-obstetric maternal mortality. The incidence has been increasing in the last two decades. ${ }^{2}$ Cardiologists and perinatologists must face a new population of patients in whom, until now, the treatment and the expectations that health professionals must face regarding mortality and long-term morbidity are beginning to be a glimpse. ${ }^{11}$

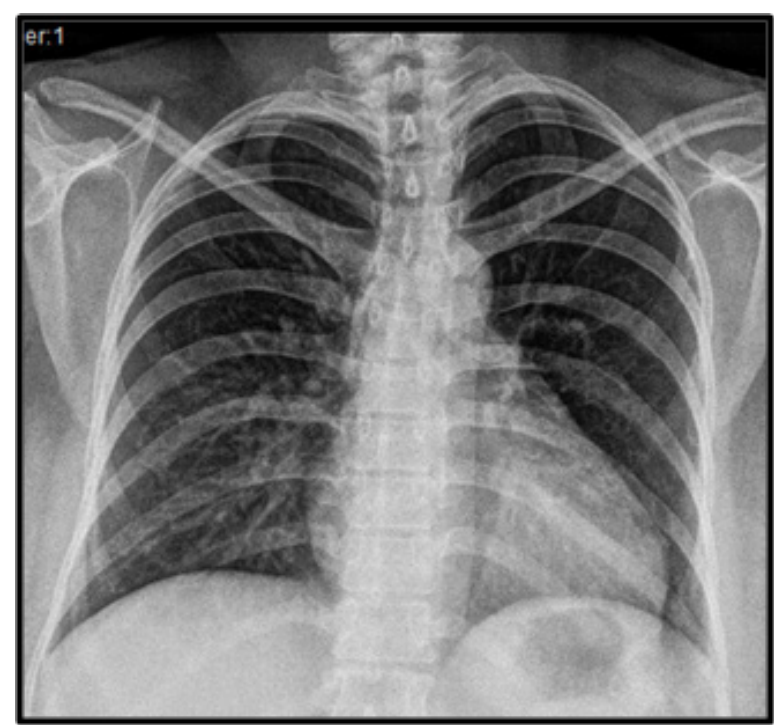

Figure 2 A.P. chest tele with a slight increase in the bronchoalveolar weft.

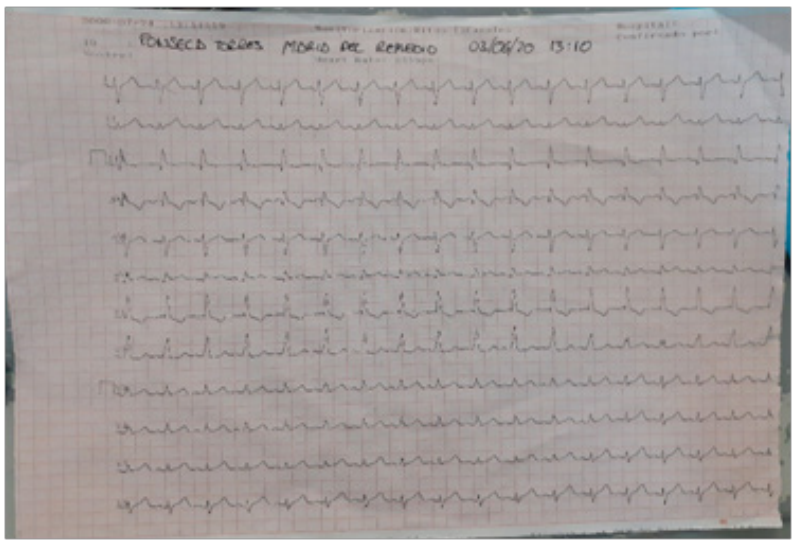

Figure 3 Electrocardiogram. Sinus rhythm, Incomplete right bundle branch block of His bundle FVM I07, and + I I0, a predominance of right forces.

This case has an uncorrected tetralogy of Fallot increase plasma volume, which led to hemodynamic pregnancy changes. The patient increased body blood volume, heart rate, and decreased pulmonary and systemic vascular resistance, causing a more magnificent short circuit from right to left heart. Oxigen saturation decreasing and cyanosis increases with hyperviscosity. ${ }^{12}$ Finally, this hemodynamics changes produce arrhythmias and thromboembolism, which are an essential cause of mortality, which is why low molecular weight heparin is essential during the puerperium.

Although the vaginal route is preferred; however, maternal cardiovascular changes have been reported to be generally less pronounced with cesarean section, and are attenuated by epidural analgesia and left lateral decubitus. If delivery is deciding, prolonged Valsalva maneuvers should be avoided during pushing since it increases afterload and decreases cardiac preload. ${ }^{13}$

Furthermore, due to the high risk of having congenital anomalies and genetic mutations, all pregnant women with congenital heart disease should undergo a fetal echocardiogram between 18 and 21 weeks of gestation. ${ }^{14}$ 

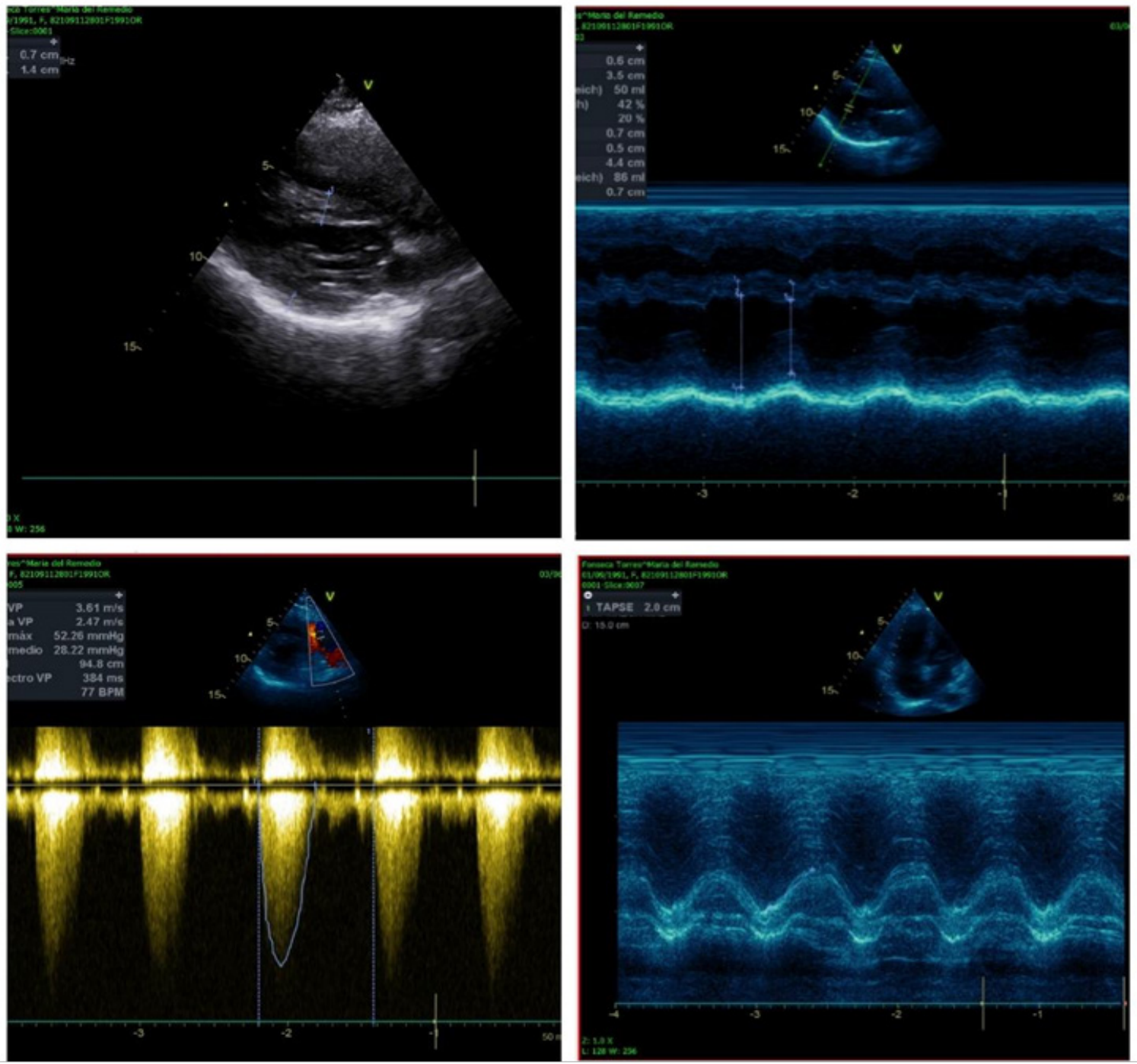

Figure 4 Echocardiogram 03-06-2020. Non-dilated non-hypertrophic left ventricle, without intracavitary thrombi, without alterations in contractility, with a moderately depressed LVEF of $42 \%$, due to teicholz, moderate diastolic dysfunction due to a type 2 pattern. Undilated right ventricle hypertrophy, without intracavitary thrombi, preserved systolic function, with TAPSE of $20 \mathrm{~mm}$, ventricular septal defect with an aortic thrust of $30 \%$, in fundibular pulmonary stenosis with a maximum gradient of $52 \mathrm{mmHg}$, medium $28 \mathrm{mmHg}$.

Table I Laboratories during their hospital stay without significant modification

\begin{tabular}{llllll}
\hline Analite/Date & $\mathbf{0 3 - 0 6 - 2 0 2 0}$ & $\mathbf{0 4 - 0 6 - 2 0 2 0}$ & $\mathbf{0 5 - 0 6 - 2 0 2 0}$ & $\mathbf{0 6 - 0 6 - 2 0 2 0}$ & $\mathbf{0 8 - 0 6 - 2 0 2 0}$ \\
\hline Glucose $(\mathrm{mg} / \mathrm{dl})$ & 64 & 91 & 75 & 77 & 74 \\
Creatinine $(\mathrm{mg} / \mathrm{dl})$ & 0.5 & 0.5 & 0.6 & 0.6 & 0.5 \\
Total Bilirrubine(mg/d) & - & - & 1.40 & 1.20 & 0.80 \\
DB (mg/dl) & - & - & 0.40 & 0.30 & 0.30 \\
IB (mg/dl) & - & - & 1.00 & 0.90 & 0.50 \\
Hemoglobine (g/dl) & 11.20 & 11.20 & 11.30 & 11.10 & 10.9 \\
Hematocrit $(\%)$ & 34 & 34 & 32.9 & 33.2 & 32.8 \\
Platelets $(\times \mid 09 / \mathrm{L})$ & 107000 & 107000 & 115000 & 118000 & \\
\hline
\end{tabular}

Citation: Salas-Villarruel SA, Pérez-Silva S, Jiménez-Báez MV, et al. Fallot tetralogy course not corrected during pregnancy. about a case. MOJ Clin Med Case Rep. 2020;10(4):89-92. DOI: 10.15406/mojcr.2020.10.0035 I 
Based on scientific evidence and clinical experience, experts have validated that the modified risk classification of the World Health Organization (WHO) has validated as the most reliable risk assessment method. Four categories are in this classification. Class, women include those with no detectable increase in maternal mortality, and with no increase or a slight increase in morbidity, in these patients, the risk is shallow. Class II includes those women with a slightly increased risk of maternal mortality or a moderate increase in morbidity. Class III represents those women with a considerably increased risk of maternal mortality or severe morbidity, and class IV includes those women with an extremely high risk of maternal mortality or severe morbidity where pregnancy should be discouraged. ${ }^{15,16}$

Presbyter et al. showed that the most critical risk factors for adverse fetal outcomes are: the degree of cyanosis, systemic oxygen saturation below $85 \%$, and hemoglobin concentrations greater than $20 \mathrm{~g} / \mathrm{dL},{ }^{17}$ which our patient did not present.

Regarding decision-making, joint treatment performed during childbirth and the puerperium by critical Medicine, Cardiology, anesthesiology, pediatrics, and gynecology, which had a significant impact on mother-child well-being.

\section{Conclusion}

Pregnancy produces significant hemodynamic effects in the patient with congenital heart defects. Anatomical structural injury calculates the level of risk for complications up to fatal outcomes for the patient and the product. The evidence available on the subject is still low due to the incidence of pregnancy in patients with corrected or uncorrected significant heart disease, so preconception consultation in this population should be privileged for risk stratification to make the best decisions regarding treatment. Emphasize that these patients should receive adequate advice from their treating doctors regarding family planning and the use of a safe and effective contraceptive method that allows them to plan their pregnancy in the best conditions.

\section{Conflict of interest}

The authors have no conflicts of interest to declare.

\section{Funding}

None.

\section{Acknowledgment}

None.

\section{References}

1. Anderson RH, Jacobs ML. The anatomy of tetralogy of Fallot with pulmonary stenosis. Cardiol Young. 2008;3:12-21.
2. Manso B, Gran F, Pijuán A, et al. Pregnancy and congenital heart disease. Rev Esp Cardiol. 2008;61(3):236-43.

3. Torres-Gómez LG, Armida Î̃nigo-Riesgo C, Espinoza-Ortegón MA, et al Embarazo y tetralogía de Fallot con y sin corrección quirúrgica. Ginecol Obst Mex. 2010;78(6):309-315.

4. Marelli AJ, Mackie AS, Ionescu-Ittu R, et al. Congenital heart disease in the general population: changing prevalence and age distribution. Circulation. 2007;115:163-172.

5. Halla ME. Georgeb EM, Grangerb JP. El corazón durante el embarazo. Rev Esp Cardiol. 2011;64 (11):1045-1050.

6. Drenthen W, Pieper PG, Roos-Hesselink. The outcome of pregnancy in women with congenital heart disease: a literature review. J Am Coll Cardiol. 2007;49(24):2303-2311.

7. López MGE, Quiroz A, Casanova JM, et al. Tetralogía de Fallot en adultos. Arch Inst Cardiol Mex. 1997;67:405-410.

8. M.G. Earing, G.D. Webb. Congenital heart disease and pregnancy: maternal and fetal risks. Clin Perinatol. 2005;32(4):913-919.

9. Hink E, Bolte AC. Pregnancy outcomes in women with heart disease: an experience of a tertiary center in the Netherlands. Pregnancy Hypertens. 2015;5(2):165-170.

10. Breitbart RE, Fyler DC. Tetralogy of Fallot. Keane: Nadas' Pediatric Cardiology, 2nd ed. Saunders. 2006;559-579.

11. Rendón Iván D, Soto Mariana, Jaramillo Mónica, et al. Tetralogía de Fallot y embarazo. Rev Colomb Cardiol. 2014;21(4):246-250.

12. Child J.S. Fallot's tetralogy and pregnancy: prognostication and prophecy. J Am Coll Cardiol. 2004;44(1):181-183.

13. Mendelson MA. Cardiopatías congénitas y embarazo. Atención perinatal de trastornos maternos crónicos. Clin Perinatología. México: McGrawHill Interamericana. 1997;2:485-500.

14. Greutmann M, Pieper PG. Pregnancy in women with congenital heart disease. Eur Heart J. 2015;36(37):2491-2499.

15. Balci A, Sollie-Szarynska KM, van der Bijl AG, et al. Prospective validation and assessment of cardiovascular and offspring risk models for pregnant women with congenital heart disease. Heart. 2010;100(17):1373-1381.

16. Thorne S, Mac Gregor A, Nelson-Piercy C. Risks of contraception and pregnancy in heart disease. Heart. 2006;92(10):1520-1525.

17. Presbitero P, Somerville J, Stone S, et al. pregnancy in cyanotic congenital heart disease. The outcome of the mother and fetus. Circulation. 1994;89(6):2673-2676. 